\title{
Hydrothermal degradation behavior of Y-TZP ceramics sintered by non-conventional microwave technology
}

\author{
Álvaro Presenda ${ }^{1}$, M. Dolores Salvador ${ }^{1}$, Rodrigo Moreno $^{2}$, Amparo Borrell $^{1^{*}}$ \\ ${ }^{1}$ Instituto de Tecnología de Materiales (ITM), Universidad Politécnica de Valencia, Camino de \\ Vera s/n, 46022, Valencia, Spain \\ ${ }^{2}$ Instituto de Cerámica y Vidrio (ICV), Consejo Superior de Investigaciones Científicas (CSIC), \\ E-28049 Madrid, Spain
}

*Corresponding author at: Instituto de Tecnología de Materiales (ITM), Universidad Politécnica de Valencia, Camino de Vera s/n, 46022, Valencia, Spain. Tel.: +34963877007; Fax: +34963 877629. E-mail address: aborrell@upv.es (A. Borrell)

Keywords: Y-TZP materials; Microwave sintering; Mechanical properties; Hydrothermal degradation; Dental applications

\begin{abstract}
Different factors such as the characteristics of starting powders, their processing, the sintering technique and the final sintering temperature were assessed with the goal to improve the low temperature degradation (LTD) resistance of 3Y-TZP materials without compromising on the mechanical properties. The degradation of hydrothermally treated specimens was studied by AFM, nanoindentation technique, micro-Raman spectroscopy and electron microscopy.

3Y-TZP previously prepared in laboratory by colloidal processing, and sintered by microwave method at low temperature $\left(1200{ }^{\circ} \mathrm{C}\right)$ led to excellent mechanical and LTD resistance, as compared to dental restorations based on Y-TZP commercial material. In the former, the presence of $m$-phase was almost non-existent even after $200 \mathrm{~h}$ of exposure to LTD conditions and the initial mechanical properties were maintained, giving $16 \mathrm{GPa}$ and $250 \mathrm{GPa}$ mean values for hardness and Young's modulus, respectively. The influence of the fast-technology by microwave heating is presented with a non-conventional sintering method to fabricate 3Y-TZP
\end{abstract}


ceramics for dental application with very high resistance against LTD and optimized mechanical properties.

\section{Introduction}

Yttria $\left(\mathrm{Y}_{2} \mathrm{O}_{3}\right)$ stabilized tetragonal zirconia polycrystalline (Y-TZP) ceramics have become significantly important as biomaterials for dental applications due to their superb mechanical properties, null interaction with human tissue and promising aesthetic characteristics [1]. Their relatively high strength, hardness and fracture toughness make them excellent candidates for dental prostheses and implants. A mechanism known as transformation toughening is responsible for the high strength and toughness of zirconia materials. This mechanism consists on the spontaneous martensitic transformation of the metastable tetragonal $(t)$ phase to the room-temperature stable monoclinic $(m)$ configuration as a crack propagates through the material. The transformed particles surround and enclose the crack inhibiting its growth [2]. Therefore, it is very important that the material is completely stabilized in the $t$-phase. In order to obtain zirconia materials with almost $100 \% t$-phase content, a stabilizing agent needs to be added. Particularly, zirconia materials for dental application contain low amounts of yttria (1.5 $3.5 \mathrm{~mol} \%)$.

However, this advantageous toughening mechanism has also become counterproductive when Y-TZP materials are exposed to humid environments at temperatures of $20-300{ }^{\circ} \mathrm{C}$, due to a hydrothermal ageing phenomenon known as low temperature degradation (LTD) [3-5]. Between the years 1999 and 2001, Y-TZP femoral heads utilized for hip replacement surgery were reported to fail catastrophically inside the human body [4,6]. In 2007, these failures were attributed to LTD and since then this hydrothermal ageing process has received a lot of attention. The conditions that favor LTD are present in the oral cavity; hence, it is extremely important to study the effect of LTD on Y-TZP materials for dental applications. Several theories have been proposed on the interaction and effects of water in LTD [7-9] but the mechanisms that trigger this phenomenon have not been truly understood yet. Nonetheless, it is well established that the degradation starts from the surface and proceeds to the material bulk inwards. The $t-m$ transformation is accompanied by a change in volume that results in the introduction of defects in the material such as surface uplifts and microcracks [10]. As a consequence, surface roughening and macrocracking arises and the mechanical and aesthetic properties are affected [11-13]. Therefore, it is extremely important to investigate the susceptibility of Y-TZP dental materials to LTD, especially when it comes to microstructure and mechanical properties. 
There are different factors that affect the LTD behavior in zirconia materials including grain shape and size, stabilizer content and distribution, cubic phase content, porosity, and effect of residual stresses [4,14-17]. Particularly, grain size and shape are strongly influenced by the sintering process and its conditions $[18,19]$. In ceramics, sintering is a common thermal treatment that allows the consolidation of dense materials from starting powders by the activation of mass transfer mechanisms and plays a significant role in the resulting microstructure and mechanical properties. Dwell time and temperature are the most important parameters that define grain size, hardness and other mechanical properties when sintering YTZP ceramics by conventional methods [20]. Conventional sintering of Y-TZP materials consists on the heating of "green" bodies at temperatures ranging from $1200-1500{ }^{\circ} \mathrm{C}$ for periods of time that may add up to several hours. The degree of densification, which is directly related to the resulting microstructure, depends on the temperature and dwell time. Heat is transferred in an industrial furnace via conventional heat transfer mechanisms: conduction, convection and radiation. This type of heating is characterized by a temperature gradient from the surface to the bulk of the material. Conventional sintering is a very energy intensive process because of the heating rates, temperatures and long times that are required.

During the past decade a strong effort was devoted to the manufacturing stage through novel near-net shaping methods. Currently, a significant part of undergoing research is focusing on innovative approaches for sintering ceramic materials. These approaches are regarded as nonconventional sintering methods. One such approach is microwave heating technology. Microwave heating consists on the absorption of electromagnetic radiation by materials due to their intrinsic dielectric properties [21,22]. It differs greatly from conventional sintering because heat transfer mechanisms are not the driving force for densification but rather the conversion of electromagnetic energy into thermal energy within the material. Therefore, the temperature gradient is created from the material bulk towards the surface. This type of heating is also known as volumetric heating [23-25]. Ceramic materials such as Y-TZP are able to absorb microwave radiation due to the presence of molecular dipoles [21]. These dipoles interact with the oscillating electric field, which is induced by the microwaves, by rotating continuously in order to align with the field. Molecular resistance to these rotations generates heat, elevating the material's temperature, a process known as dielectric heating [26]. The rise in temperature is determined by the amount of energy absorbed in the process.

Several authors [27-30] have determined that highly dense materials without substantial grain coarsening may be obtained with microwave sintering since dwell times are considerably shortened and heating rates can be substantially increased. In some studies, lower sintering temperatures have been applied while obtaining materials with relative densities comparable to those of conventional sintering at higher temperatures [30]. As Y-TZP materials with a finer 
microstructure and a high degree of densification can be obtained, mechanical properties may be enhanced, improving the overall quality of the resulting material [29]. The modified microstructure that results from microwave sintering is very likely to affect the behavior of $\mathrm{Y}$ TZP materials against hydrothermal degradation. Moreover, shortening dwell times and lowering sintering temperatures reduce the amount of energy consumption during the process, which may also translate in lower production costs for professionals and patients. Other advantages include a lower environmental impact than other sintering techniques and the possibility to process near-net-shape materials. Therefore, microwave sintering and its advantages represent a novel and interesting alternative for sintering Y-TZP dental materials.

Up to now, several studies have focused on the understanding of the LTD process and its effect on Y-TZP dental materials. Chevalier et al. [3,4], Vluegels et al. [31], and Cattani et al. [32] have carried out in-depth investigations on the ageing process of zirconia, such as $m$-phase content evolution, degradation of mechanical properties and surface roughening. The influence of processing conditions, addition of other compounds such as $\mathrm{Al}_{2} \mathrm{O}_{3}$, and $\mathrm{Y}_{2} \mathrm{O}_{3}$-stabilizer incorporation on LTD has also been explored for dental materials sintered via conventional methods $[18,19,33]$. However, no comprehensive study has been carried out regarding the influence of microwave sintering on Y-TZP materials exposed to LTD environments. Since the sintering process and its conditions are a critical factor in determining the LTD behavior of YTZP materials, it is very important to investigate the effect of microwave sintering techniques on this phenomenon.

The purpose of this study is to evaluate the effect of microwave sintering on LTD susceptibility of dental restorations based on Y-TZP material by comparing them with materials sintered by the conventional method. Surface roughening, mechanical properties such as hardness and Young's modulus, microstructure and $m$-phase transformation progression are assessed as a function of degradation time under simulated conditions. The blank hypothesis to be tested in this work is that microwave-sintering method has a great influence on the LTD behavior of YTZP dental materials.

\section{Materials and methods}

Two Y-TZP materials stabilized with $3 \mathrm{~mol} \% \mathrm{Y}_{2} \mathrm{O}_{3}$ were employed in this work. One of them is a commercial yttria-stabilized zirconia dental material provided as a pre-sintered block (LAVA Zirconia, 3M ESPE, St. Paul, MN, USA). According to the manufacturer's specifications sheet, this zirconia material has been prepared as a co-precipitated, spray-dried powder with a particle size range between $0.07-0.3 \mu \mathrm{m}$. Samples have been cut from the pre-sintered block and shaped into $10 \mathrm{~mm}$-diameter cylinders. The second one is modified Y-TZP powder (TZ3YS, 
TOSOH, Japan), which has been prepared in the laboratory by colloidal processing optimizing its rheological behavior, thus resulting in a more homogeneous material in terms of particle size than the commercial materials. The average particle size of this powder is approximately 100 $\mathrm{nm}$ and a specific surface area of $14.5 \mathrm{~m}^{2} \cdot \mathrm{g}^{-1}$. For a description of the preparation process of this powder see Reference 35 . Those powders were pelletized by uniaxial compression at a pressure load of $50 \mathrm{MPa}$ to obtain discs with $10 \mathrm{~mm}$ in diameter that were used for sintering tests.

\section{Samples sintering}

Conventional sintering (CS) was performed in an electrical furnace (Thermolyne type 46100, Thermo Fisher Scientific, USA) in air at $1400{ }^{\circ} \mathrm{C}$ with a $10^{\circ} \mathrm{C} \cdot \mathrm{min}^{-1}$ heating rate and $2 \mathrm{~h}$ of dwell time. Microwave (MW) heating technology has been employed as a non-conventional sintering technique. In this case, samples were introduced in a mono-mode $(2.45 \mathrm{GHz})$ rectangular cavity that is automatically adjusted to optimize microwave absorption and control the heating rate of $100{ }^{\circ} \mathrm{C} \cdot \mathrm{min}^{-1}$. The final sintering temperature of $1200^{\circ} \mathrm{C}$ and holding time of 10 min were selected. These selected parameters are based on previous studies in our research group, where sintering conditions were optimized for Y-TZP materials [29,35,36]. The nomenclature of the samples was defined as follows: LAVA-MW and LAVA-CS for LAVA material, and LAB-MW and LAB-CS for the lab-prepared Tosoh powder.

\section{Characterization of as-sintered and hydrothermally aged samples}

Characterization of as-sintered (AS) and aged samples consists of phase content analysis, surface topography and roughness, evaluation of mechanical properties, and microstructure assessment. Low temperature degradation (LTD) of Y-TZP materials is carried out under conditions that accelerate the hydrothermal ageing process. Specimens are mirror polished and autoclaved in steam at $125^{\circ} \mathrm{C}$ and 1.6 bar. Characterization of aged samples is performed after every $20 \mathrm{~h}$ of exposure to LTD conditions until $200 \mathrm{~h}$ treatment is reached.

Phase content analysis was performed with a micro-Raman spectrometer (LabRam HR UV, HORIBA Jobin Yvon, France) coupled with a thermoelectrically-cooled multichannel CCD detector. Measurements were carried out on the exposed, polished surface. Raman spectra were recorded for a Raman shift range of $120-700 \mathrm{~cm}^{-1}$ as an average of two successive measurements, each with an integration time of $2 \mathrm{~min}$, in order to obtain a well-defined spectrum. A laser wavelength of $532 \mathrm{~nm}$ through a 50x objective with a lateral resolution of approximately $2 \mu \mathrm{m}$ was employed. Monoclinic phase content in aged specimens is determined with the following linear model proposed by Lim et al. [37], where $V_{m}$ represents the $m$-phase volume fraction: 
$V_{m}=\frac{I_{m}^{181}+I_{m}^{190}}{0.33\left(I_{t}^{147}+I_{t}^{265}\right)+I_{m}^{181}+I_{m}^{190}}$

According to Muñoz-Tabares and Anglada [38], this equation provides the lowest average absolute error between real and calculated $V_{m}$ among several models utilized for quantification of $m$-phase zirconia content. Peak intensities, $I$, of the characteristic $t$-phase bands at 147 and $265 \mathrm{~cm}^{-1}$ and $m$-phase bands at 181 and $190 \mathrm{~cm}^{-1}$ have been quantified with the spectroscopic analysis function in Origin 8 software. A lineal background signal, which was subtracted, has been assumed, while the integrated intensity of each peak has been calculated by fitting Lorentzian distribution curves to the spectrum.

Surface topography and roughening were analyzed by means of atomic force microscopy (AFM) in tapping mode (Multimode, Veeco, USA). Two topographic images have been taken for each sample: one with a $1 \times 1 \mu \mathrm{m}$ scan area to have a detailed view of grains and another with a $5 \times 5 \mu \mathrm{m}$ scan area in order to provide a representative roughness value. A doped silicon probe of $10 \mathrm{~nm}$ tip radius has been utilized. Image processing and roughness calculations are performed with Nanoscope Analysis software. Nomarski microscopy (Eclipse LV100, Nikon, Japan) has also been employed to assess surface topography after each degradation phase.

To estimate the depth of penetration of the $m$-transformed zone after hydrothermal treatment samples were cross-sectioned, polished, and analyzed with a field-emission scanning electron microscope (FE-SEM, S4800, Hitachi, SCSIE of the University of Valencia).

Regarding mechanical properties, hardness, $H$, and Young Modulus, $E$, were evaluated via the nanoindetation technique. The system utilized in this work consists of a nanoindenter (G-200, Agilent Technologies, USA) with a Berkovich tip previously calibrated with silica standard. Tests were performed under maximum depth control of the $2000 \mathrm{~nm}$. The contact stiffness (S) was determined by Continuous Stiffness Measurement technique (CSM) to calculate the profiles of hardness $(H)$ and elastic modulus $(E)$ [39]. Amplitude was programmed to $2 \mathrm{~nm}$ with $45 \mathrm{~Hz}$ of frequency. A matrix with 25 indentations was performed for each sample.

\section{Results and Discussion}

As-sintered samples

Relative density measurements have been performed in all as-sintered (AS) samples in order to determine whether a sufficiently high degree of densification has been achieved with the selected sintering conditions so the samples can be effectively compared. Mechanical properties 
of the AS samples also serve as important parameters to validate and adequately compare the progression of LTD because, even though different sintering methods were employed. Table 1 shows sintering parameters and the most relevant properties for AS samples.

Regarding surface topography $\left(\mathrm{R}_{\mathrm{a}}\right)$, all AS samples have been carefully polished with colloidal silica resulting in roughness values ranging from $1-3 \mathrm{~nm}$. In this manner, the surface has been prepared for assessing the changes that arise in surface topography once LTD tests of the materials are carried out.

Mechanical properties for these samples fall within an acceptable range for fully dense Y-TZP materials (240 - 270 GPa for Young's modulus, $E$, and $15-17 \mathrm{GPa}$ for hardness, H) [1]. E and $H$ values of LAVA materials tend to be lower than their LAB counterparts. The lower roughness values and better mechanical properties in $\mathrm{LAB}$ material samples are due to the fact that LAB has been carefully prepared with a previous rheological study that optimizes the characteristics of the starting material. On the other hand, LAVA material has a commercial nature and, therefore, we could not control the initial processing, this aspect must be taken into consideration.

Microstructural features such as grain size and shape play a very important role in LTD behavior of Y-TZP ceramic materials. Therefore, a thorough characterization of the microstructure of AS samples was performed by means of FE-SEM (Figure 1). Samples that were sintered via microwave heating shows some interesting differences between each other, which is expected due to shorter dwell times and higher heating rates. As can be observed in the micrographs (Figure 1a and 1b), microwave sintered material shows a finer microstructure than conventionally sintered material. In case of LAVA-MW material, a wide range of grain sizes is observed, from very fine grains less than $50 \mathrm{~nm}$ to particles larger than $210 \mathrm{~nm}$. There are also more variations in grain shape as they tend to be less rounded than their LAB counterparts. According to these images, LAB seems to provide a more homogeneous microstructure and shows a similar grain size in both cases (MW and CS). Again, this can be attributed to the characteristics of the starting material that have been optimized thanks to the previous rheological study that it underwent. The high degree of densification in microwave and conventional sintering is also reflected in the microstructure as no porosity is observed in any case.

\section{Phase composition}

Figure 2 shows Raman spectra for the LAVA-MW and LAB-MW samples, where no $m$-peaks are observed in AS material and even after $20 \mathrm{~h}$ of exposure to the given LTD conditions. 
Nonetheless, there is the possibility that a low presence of the cubic (c) phase has not been clearly revealed due to peak overlapping of $c$ and $t$ phases around 280 and $320 \mathrm{~cm}^{-1}$. As the LAVA-MW accumulated more time of exposure to LTD, the intensity of $m$-peaks increased. At $40 \mathrm{~h}, m$-peaks can be distinguished. At $200 \mathrm{~h}, t$-phase peaks are almost non-existent. However, the LAB-MW spectrum showed no $m$-peaks even after $200 \mathrm{~h}$ of LTD exposure, as can be seen in Figure 2b. LAVA-CS and LAB-CS samples show a similar trend than LAVA-MW specimens, with a gradual increase of the intensity of m-phase peaks.

The sintering method and type of material had an important influence on the volume fraction of $m$-phase, $V_{m}$, calculated from the intensities of the Raman spectra, as it progressed at different rates in all four samples, which can be seen in Figure 3. In LAVA material, the microwavesintered sample degraded slower before $100 \mathrm{~h}$ compared to the conventionally sintered sample. At $100 \mathrm{~h}$, the $V_{m}$ of both LAVA samples appear to stabilize and reach a maximum $V_{m}$ value of $90 \%$ at $140 \mathrm{~h}$. In the case of the LAB-material, the microwave-sintered sample shows no susceptibility to LTD, while the conventionally sintered sample is definitely affected by degradation, as it already contains a $V_{m}$ of $28 \%$ after $40 \mathrm{~h}$ of hydrothermal degradation exposure, despite, that both samples have a similar properties and microstructure. If conventionally sintered samples are compared between each other, LAVA material shows a higher susceptibility to $m$-phase transformation than LAB material. When comparing microwave sintered specimens, the LTD behavior of LAVA material is quite different from LAB. LAVA is substantially affected by aging, while LAB is not affected at all, even though they were sintered under the same conditions. Nonetheless, LAVA-MW still shows a higher resistance to LTD at exposure times below $100 \mathrm{~h}$ than its conventionally sintered counterpart.

The $V m$ saturation limit in both LAVA materials and LAB-CS is reached after $100 \mathrm{~h}$ at approximately, from 100 to $200 \mathrm{~h}$ does not vary significantly. The reason for not reaching $100 \%$ transformation may be attributed to the presence of the non-transformable $c$-phase in the AS material. However, no $c$-phase analysis has been carried out to determine its presence and amount.

\section{Topography and surface roughening}

When Y-TZP materials undergo the phase transformation from tetragonal to monoclinic, it is accompanied by an increase in volume of about $3-4 \%$. In order to make-up for the volume increase that accompanies the transformation, grains are pushed towards the surface, where less space constraints are found, modifying the topography of the material, which is reflected on the 
surface as roughening. In order to analyze superficial changes induced by phase transformation, AFM techniques have been employed to characterize the initial hydrothermal aging process of the samples. The mean surface roughness, $R_{a}$, has also been determined as a way of quantifying this change. As an example, Figure 4 shows AFM images of the surface of the LAB-MW and LAB-CS samples at different degradation times. After $40 \mathrm{~h}$ of LTD exposure, transformed zones can already be observed. As exposure time is increased, the topography becomes more irregular and surface roughening increases.

It is important to consider that AFM can only detect if transformation has occurred. However, it is not able to determine the amount of $m$-phase that has been transformed, which means that, even if substantial roughening can be observed, it does not imply that the surface has been $100 \%$ transformed. Nonetheless, AFM serves as a complimentary tool for assessing the phase transformation at the initial stages. This is particularly important when describing the topographical changes in the LAB-MW sample because even though no $m$-phase peaks have been detected with Raman spectroscopy, AFM indicates that already after $40 \mathrm{~h}$ of LTD exposure changes in surface topography have occurred.

In order to compare the $R_{\mathrm{a}}$ values of materials obtained with both sintering methods and treated to different ageing times, the roughness values have been determined and presented in Figure 5. Microwave sintered LAB zirconia has clearly resulted in the lowest roughening values when exposed to LTD conditions at all times. The other samples show bigger variations in roughness, especially after the first $20 \mathrm{~h}$. For example, LAB-CS shows a gradual increase in surface roughening from $1 \mathrm{~nm}$ until a value of approximately $5 \mathrm{~nm}$ is reached after $60 \mathrm{~h}$. This behavior indicates that the most significant topographical changes occur during the initial stages. Even though the $R_{a}$ of AS ( $\left.0 \mathrm{~h}\right)$ samples is not set at the same initial value, the change in $R_{a}$ after each degradation stage gives important information on how LTD affects the samples after each stage.

\section{Microstructure and penetration depth}

Analysis at a microscopic scale of the degraded surfaces has been performed by means of Nomarski optical microscopy. Figure 6 shows images of the exposed surfaces after $140 \mathrm{~h}$ of hydrothermal degradation. The effects of degradation appear as surface roughening and are clearly visible in the conventionally sintered samples (Figures $6 \mathrm{~b}$ and $6 \mathrm{~d}$ ), as well as the microwave sintered commercial material (Figure 6a). LAVA-MW appears to have the most degraded surface, as a very irregular topography can be observed. The surface of LAB-CS shows very interesting bubble-like features that are not observed in the other samples. LABMW still holds a flat smooth surface, as there has not been a significant degradation of this sample, which is in agreement with the results obtained from other characterization techniques. 
In order to determine the penetration depth of the zirconia-transformed layer after $140 \mathrm{~h}, \mathrm{FE}-$ SEM images of transversally cut samples have been obtained and are shown in Figure 7. The layer observed in the micrographs is a result of the grain detachment of the transformed zone that occurs during the polishing stage of sample preparation. Grains in the degraded zone that have expanded due to the volume change induced by phase transformation are pulled off from the material during the abrasive treatment resulting in an increase of porosity in this zone.

As can be seen in the micrographs, the penetration depth of the transformed zone varies significantly depending on the material and the sintering method. The depth of the degraded zone in LAVA-MW and LAVA-CS (Figure 7a and Figure 7b, respectively) is approximately 30 - $40 \mu \mathrm{m}$. However, the degraded layer in LAVA-MW does not seem uniform, as depths between a range of 24 and $40 \mu \mathrm{m}$ have also been measured. LAVA-CS results in a more uniform degradation layer, with a depth of approximately $30 \mu \mathrm{m}$ after $140 \mathrm{~h}$. In LAB material, the microwave-sintered sample shows a significantly higher resistance to degradation penetration than the conventionally sintered sample.

LAVA material clearly shows transformed zones progressing deeper into the material bulk than LAB material. Microwave sintered samples result in very different penetration depths after 140 $\mathrm{h}$ of LTD. Even though the results from Raman spectroscopy indicate the absence of $m$-phase zirconia in LAB-MW material, the FE-SEM image indicates a very slight, sub-superficial degraded zone of less than $5 \mu \mathrm{m}$.

\section{Mechanical properties}

As a mean for assessing the quality of the material after exposure to LTD, two important mechanical properties, $E$ and $H$, have been determined as a function of hydrothermal degradation time. These properties are affected by the sintering method that has been employed and the zirconia materials used. The results are summarized in Figure 8.

These results corroborate the high resistance to LTD of the microwave sintered LAB zirconia as $E$ and $H$ remain almost unaffected. Even after $140 \mathrm{~h}, E$ is still around $250 \mathrm{GPa}$ and $H$ is approximately $15 \mathrm{GPa}$. The properties of the other sintered samples are, however, notably degraded as hydrothermal exposure time progresses. After $20 \mathrm{~h}$, the values of $E$ and $H$ of the conventionally sintered samples are similar than for the LAVA-MW sample and remains so until $80 \mathrm{~h}$ has been reached, after LAVA-MW sample is what has the worst mechanical behaviour. When $80 \mathrm{~h}$ is reached, the $E$ values have decayed almost one-third from the AS value. This suggests that a decrease of mechanical properties may occur faster during the first 
degradation stages. In both materials sintered by the conventional method, the values of $E$ and $H$ stabilize at approximately $180 \mathrm{GPa}$ and $10 \mathrm{GPa}$, respectively.

In order to correctly interpret the values of $E$ and $H$ that have been calculated, it is important to consider the depth of penetration of the transformed zone (Figure 9). As previously stated, the indenter penetration depth was set at $2 \mu \mathrm{m}$ approximately. Therefore, during the initial degradation stages, where it is very likely that a combination of tetragonal and monoclinic zirconia, can be found at such sub-superficial depth, the $E$ and $H$ values that have been determined depend on the amount of transformed zirconia and result from a combination of both phases. For example, Figure 9 shows the depth profile of the Young's modulus in LAB-CS and LAB-MW materials, where different LTD times are represented. As can be seen, the initial degradation stages (40 and $60 \mathrm{~h}$ ) result in lower $E$ values at sub-superficial depths less than 800 $\mathrm{nm}$. However, as the indenter penetrates deeper into the material, $E$ increases because the presence of $m$-zirconia decreases. This behavior is expected as phase transformation begins on the surface and progresses into the material bulk. At $140 \mathrm{~h}$, the value of $E$ stabilized throughout the whole penetration range of $2 \mu \mathrm{m}$ indicating that, after such a long LTD exposure time, all the $t$-phase zirconia at this depth has been transformed.

\section{LTD behavior of microwave and conventionally sintered materials}

The mechanical properties obtained from microwave and conventional sintering of the AS zirconia samples demonstrate that the selected conditions in each case resulted in highly densified materials with excellent Young modulus and hardness values. Moreover, microRaman spectroscopy of the AS materials has served to determine that no presence of $m$-phase is present before exposure to LTD. Smooth and uniform surfaces have also been obtained after sample preparation in order to adequately assess the topographical changes induced by the initial stages of LTD.

The results obtained by the different characterization techniques have allowed studying the effects of LTD in the zirconia materials considered in this work. The tetragonal to monoclinic phase transformation that zirconia materials depending on the sintering method, as well as on the characteristics of the starting material. Mechanical properties have also been affected in such a way that when LTD exposure time is increased, $E$ and $H$ values decrease. Such effect on mechanical properties is extremely relevant since their high Young modulus and hardness values are one of the main reasons Y-TZP materials are considered for odontological applications. 
The different behaviors against hydrothermal degradation that have been observed in the Y-TZP material considered in this work respond to two fundamental aspects that directly affect the resulting microstructure: 1) sintering method and its conditions, and 2) characteristics of the YTZP starting material. With respect to the sintering method, microwave sintering allows the consolidation of materials with a finer particle size since dwell times are considerably shortened when compared to conventional sintering (10 vs $120 \mathrm{~min}$, respectively). Also, heating rates employed during microwave sintering are significantly higher (100 vs $\left.10^{\circ} \mathrm{C} / \mathrm{min}\right)$ and final temperatures are lower (1200 vs $1400{ }^{\circ} \mathrm{C}$ ) than in conventional sintering, which inhibit grain coarsening. Finer microstructures obtained via microwave sintering have resulted in improvement of mechanical properties as reported elsewhere [29,30,34]. Furthermore, other sources $[18,40-42]$ have established that reducing the average grain size enhances the resistance against hydrothermal degradation of Y-TZP materials because reducing the particle size increases the ratio between surface area and volume and, hence, the effectiveness of the interphase to constrain the change in shape that accompanies the transformation [43]. An alternative approach indicates that the monoclinic variants that may be accommodated when a grain is transformed decrease with its diameter [44].

In this work, particularly in the lab-prepared material obtained by microwave sintering, LTD resistance is very high since no detectable $m$-phase transformation has occurred and mechanical properties are maintained even after $200 \mathrm{~h}$ of LTD exposure. In the case of LAVA material, the susceptibility to LTD in the initial stages has been decreased, as $m$-phase content is lower and mechanical properties are higher at LTD exposure times less than $80 \mathrm{~h}$ compared to its conventionally sintered counterpart. However, at higher degradation times, a significant amount of $m$-phase content was found and an important loss of mechanical properties occurred. The differences between the commercial and lab-prepared material may be attributed to the characteristics of the starting material. Besides, other important factors may also have an influence on the hydrothermal degradation behavior such as $\mathrm{Y}_{2} \mathrm{O}_{3}$ distribution and $c$-phase content of the starting material $[18,19]$.

Another important aspect to consider in the variation of degradation kinetics between conventional and microwave sintering is the final temperature that has been employed for each method. Inokoshi et al. [18] and Gaillard et al. [45] have demonstrated that higher sintering temperatures increase the content of $c$-phase in accordance with the zirconia phase diagram. The presence of cubic zirconia accelerates the transformation of tetragonal to monoclinic phase, as tetragonal grains are depleted from $\mathrm{Y}_{2} \mathrm{O}_{3}$ due to the migration of the stabilizer into cubic grains $[5,16]$.

As microwave sintering requires lower temperatures for the full consolidation of Y-TZP ceramics than conventional sintering, the amount of c-phase content is reduced and the 
resistance to LTD is enhanced. This idea may also serve to explain the higher and interesting resistance to LTD of the specimens sintered via microwaves at $1200{ }^{\circ} \mathrm{C}$ against conventional sintered ones at $1400^{\circ} \mathrm{C}$. However, it is difficult to conclude whether the content of c-phase has been substantially increased at $1400{ }^{\circ} \mathrm{C}$ since no c-phase content analysis was performed on the AS materials.

Another important factor to fabricate Y-TZP materials by non-conventional microwave technique for odontological applications is the fact to obtain this material of a white color, because the other non-conventional spark plasma sintering technique (SPS) has a big problem with the sintering of zirconia materials [30]. By SPS the zirconia sintered shows a full black color. This is due to carbon diffusion within the zirconia sample by SPS processing, which is linked to the carbon rich atmosphere in which it is performed. As the sintering of the compact is taking place in a graphite die, the carbon diffuses into the sample from the die and this process is promoted by the applied pressure.

\section{Conclusions}

The blank hypothesis initially established in this work that microwave sintering had effect in the behavior of Y-TZP materials against LTD has been confirmed. Microwave sintering has a significant influence on the resulting microstructure of Y-TZP sintered ceramics and, hence, on their hydrothermal degradation susceptibility. Fully-dense materials may be obtained via microwave heating technology and at lower sintering temperatures than with the conventional method, resulting in a finer microstructure. This microstructure may provide a higher resistance to LTD. The LAB material sintered via microwave, the presence of $m$-phase was almost nonexistent even after $200 \mathrm{~h}$ of exposure to LTD conditions and the initial mechanical properties have been maintained. By combining a carefully prepared starting material with a homogeneous particle size and microwave heating technology as the sintering method, Y-TZP ceramics for odontological application with very high resistance against LTD may be obtained. In the commercial material, hydrothermal degradation was slowed down at the initial stages. Other factors that influence the behavior against LTD, such as $c$-phase content and type, amount and distribution of stabilizer and the effects that microwave sintering mechanism could have in them need to be further studied. There is not previous works published regarding the influence of microwave sintering on LTD, therefore this work is a approach to know the LTD behavior of dental materials obtained by non-conventional microwave method. 


\section{Acknowledgements}

A. Presenda acknowledges the Generalitat Valenciana for his Santiago Grisolía scholarship (GRISOLIA/2013/035). A. Borrell acknowledges the Spanish Ministry of Science and Innovation for her Juan de la Cierva contract (JCI-2011-10498) and the Generalitat Valenciana for the financial support under the GV/2014/009 project. M. D. Salvador thanks CAPES Programa Ciências sem Fronteiras (Brazil) for the concession of a PVE project No A086/2013.

\section{References}

[1] Piconi C, Maccauro G. Zirconia as a ceramic biomaterial. Biomat 1999;20:1-25.

[2] McMeeking RM, Evans AG. Mechanics of transformation-toughening in brittle materials. J Am Ceram Soc 1982;65:242-6.

[3] Chevalier J, Cales B, Drouin JM. Low-temperature aging of Y-TZP ceramics. J Am Ceram Soc 1999;82:2150-4.

[4] Chevalier J, Gremillard L, Deville S. Low-temperature degradation of zirconia and implications for biomedical implants. Annu Rev Mater Res 2007;37:1-32.

[5] Lawson S. Environmental degradation of zirconia ceramics. J Eur Ceram Soc 1995;15:485502.

[6] Norton MR, Yarlagadda R, Anderson GH. Catastrophic failure of the ElitePlus total hip replacement, with a hylamer acetabulum and zirconia ceramic femoral head. J Bone Joint Surg Br 2002;84(5):631-5.

[7] Sato T, Shimada M. Crystalline phase change in yttria-partially-stabilized zirconia by lowtemperature annealing. J Am Ceram Soc 1984;67:212-23.

[8] Lange FF, Dunlop GL, Davis BL. Degradation during ageing of transformation-toughened $\mathrm{ZrO}_{2}-\mathrm{Y}_{2} \mathrm{O}_{3}$ materials at $250{ }^{\circ} \mathrm{C}$. J Am Ceram Soc 1986;69:237-40.

[9] Guo X. Hydrothermal degradation mechanism of tetragonal zirconia. J Mater Sci 2001;36:3737-44.

[10] Lughi V, Sergo V. Low temperature degradation -aging- of zirconia: A critical review of the relevant aspects in dentistry. Dent Mater 2010;26(8):807-20.

[11] Roy ME, Whiteside LA, Katerberg BJ, Steiger JA. Phase transformation, roughness, and micro-hardness of artificially aged yttria and magnesia-stabilized zirconia femoral heads. J Biomed Mater Res A 2007;83(4):1096-102. 
[12] Flinn BD, deGroot DA, Mancl LA, Raigrodski AJ. Accelerated aging characteristics of three yttria-stabilized tetragonal zirconia polycrystalline dental materials. J Prosthet Dent 2012;108(4):223-30.

[13] Ban S, Sato H, Suehiro Y, Nakanishi H, Nawa M. Biaxial flexure strength and low temperature degradation of $\mathrm{Ce}-\mathrm{TZP} / \mathrm{Al}_{2} \mathrm{O}_{3}$ nanocomposite and Y-TZP as dental restoratives. $\mathrm{J}$ Biomed Mater Res B 2008;87B(2):492-8.

[14] Lange FF. Transformation toughening Part 1: Size effects associated with the thermodynamics of constrained transformations. J Mater Sci 1982;17: 225-34

[15] Wang J, Stevens R. Surface toughening of TZP ceramics by low temperature ageing. Ceram Int 1989;15:15-21

[16] Chevalier J, Deville S, Munch E, Jullian R, Lair F. Critical effect of cubic phase on aging in $3 \mathrm{~mol} \%$ yttria-stabilized zirconia ceramics for hip replacement prosthesis. Biomat 2004;25:5539-45.

[17] Schmauder S, Schubert H. Significance of internal stresses for the martensitic transformation in yttria-stabilized tetragonal zirconia polycrystals during degradation. J Am Ceram Soc 1986;69(7):534-40.

[18] Inokoshi M, Zhang F, Vleugels J, Vanmeensel K. Influence of sintering conditions on lowtemperature degradation of dental zirconia. Dent Mater 2014;30:669-78.

[19] Zhang F, Van Meensel K, Inokoshi M, Batuk M, Hadermann J, Van Meerbeek B, Naert I, Vleugels J. 3Y-TZP ceramics with improved hydrothermal degradation resistance and fracture toughness. J Eur Ceram Soc 2014;34:2453-63.

[20] Anselmi-Tamburini U, Garay JE, Munir ZA. Fast low-temperature consolidation of bulk nanometric-ceramic materials. Scripta Mater 2006;54:823-8

[21] Goldstein A, Travitzky N, Singurindi A, Kravchik M. Direct microwave sintering of yttriastabilized zirconia at 2.45 GHz. J Eur Ceram Soc 1999;19:2067-74.

[22] Bodhak S, Bose S, Bandyopadhyay A. Densification study and mechanical properties of microwave-sintered mullite and mullite-zirconia composites. J Am Ceram Soc 2010;94:32-41

[23] Upadhaya DD, Ghosh A, Gurumurthy KR, Prasad R. Microwave sintering of cubic zirconia. Ceram Int 2001;27:415-8.

[24] Clark DE, Folz DC, West JK. Processing materials with microwave energy. Mater Sci Eng A $2000 ; 287: 153-8$. 
[25] Binner J, Annapoorani K, Paul A, Santacruz I, Vaidhyanathan B. Dense nanostructured zirconia by two-stage conventional/hybrid microwave sintering. J Eur Ceram Soc 2008;28:9737.

[26] Thostenson ET, Chou TW. Microwave processing: fundamentals and applications. Compos Part A-Appl S 1999;30:1055-71.

[27] Park SS, Meek TT. Characterization of $\mathrm{ZrO}_{2}-\mathrm{Al}_{2} \mathrm{O}_{3}$ composites sintered in a $2.45 \mathrm{GHz}$ electromagnetic field. J Mater Sci 1991;26:6309-13.

[28] Oghbaei M, Mirzaee O. Microwave versus conventional sintering: a review of fundamentals, advantages and applications. J Alloy and Compd 2010;494:175-89.

[29] Borrell A, Salvador MD, Peñaranda-Foix F, Catalá-Civera JM. Microwave sintering of zirconia materials: mechanical and microstructural properties. Int J Appl Ceram Tec 2013;10:313-20.

[30] Borrell A, Salvador MD, Rayón E, Peñaranda-Foix FL. Improvement of microstructural properties of 3Y-TZP materials by conventional and non-conventional sintering techniques. Ceram Int 2012;38:39-43.

[31] Vleugels J, Yuan ZX, Van der Biest O. Mechanical properties of $\mathrm{Y}_{2} \mathrm{O}_{3} / \mathrm{Al}_{2} \mathrm{O}_{3}$-coated $\mathrm{Y}$ TZP ceramics. J Eur Ceram Soc 2002; 22:873-81.

[32] Cattani-Lorente M, Scherrer SS, Ammann P, Jobin M, Wiskott A. Low temperature degradation of a Y-TZP dental ceramic. Acta Biomater 2011;7:858-65.

[33] Zhang F, Van Meensel K, Inokoshi M, Batuk M, Hadermann J, Van Meerbeek B, Naert I, Vleugels J. Critical influence of alumina content on the low temperature degradation of 2-3 mol\% yttria-stabilized TZP for dental restorations. J Eur Ceram Soc 2015;35(2):741-50.

[34] Vasudevan R, Karthik T, Ganesan S, Jayavel R. Effect of microwave sintering on the structural and densification behavior of sol-gel derived zirconia toughened alumina (ZTA) nanocomposites. Ceram Int 2013;39:3195-204.

[35] Rayón E, Moreno R, Alcázar C, Salvador MD, Manjón FJ, Jiménez-Piqué E, Llanes L. Enhanced hydrothermal resistance of Y-TZP ceramics through colloidal processing. J Am Ceram Soc 2013;96(4):1070-6.

[36] Benavente R, Salvador MD, Alcázar MC, Moreno R. Dense nanostructured zirconia compacts obtained by colloidal filtration of binary mixtures. Ceram Int 2012;38:2111-7.

[37] Lim C, Finlayson T, Ninio F, Griffiths J. In-situ measurement s of the stress-induced phase transformation in magnesia-partially-stabilized zirconia using Raman spectroscopy. J Am Ceram Soc 1992;75:1570-3. 
[38] Muñoz-Tabares JA, Anglada M. Quantitative analysis of monoclinic phase in Y-TZP by Raman spectroscopy. J Am Ceram Soc 2010;93(6):1790-5.

[39] Oliver WC, Pharr GM. An improved technique for determining hardness and elastic modulus using load and displacement sensing indentation experiments. J Mater Res 1992;19:1564-83.

[40] Jue J, Chen J, Virkar A. Low-temperature aging of t-zirconia: The role of microstructure on phase stability. J Am Ceram Soc 1991;74:1811-20.

[41] Gremillard L, Chevalier J, Epicier T, Deville S, Fantozzi G. Modelling the aging kinetics of zirconia. J Eur Ceram Soc 2004;24:3483-9.

[42] Li J, Watanabe R. Phase transformation in $\mathrm{Y}_{2} \mathrm{O}_{3}$-partially-stabilized $\mathrm{ZrO}_{2}$ polycrystals of various grain sizes during low-temperature aging in water. J Am Ceram Soc 1998;81:2687-91.

[43] Jeong WC, Matlock DK, Krauss G. Observation of deformation and transformation behavior of retained austenite in a $0.14 \mathrm{C}-1.2 \mathrm{Si}-1.5 \mathrm{Mn}$ steel with ferrite-bainite-austenite structure. Mater Sci Eng A 1993;165(1):1-8.

[44] Evans AG, Burlingname N, Drory M, Kriven WM. Martensitic transformation in zirconiaparticle size effects and toughening. Acta Metall 1981;29:447-56.

[45] Gaillard Y, Jiménez-Piqué E, Soldera F, Mücklich F, Anglada M. Quantification of hydrothermal degradation in zirconia by nanoindentation. Acta Mater 2008;56:4206-16. 


\section{Figure captions:}

Figure 1. FE-SEM micrographs of AS samples for a) LAVA-MW, b) LAVA-CS, c) LAB-MW, and d) LAB-CS.

Figure 2. Raman spectra obtained for a) LAVA-MW and b) LAB-MW at various hydrothermal degradation times.

Figure 3. Monoclinic phase volume content as a function of hydrothermal degradation exposure time for all samples.

Figure 4. Topographical AFM images of the exposed surface at several LTD exposure times for LAB-MW: a) AS, c) $40 \mathrm{~h}$ and e) $140 \mathrm{~h}$, and LAB-CS: b) AS, d) $40 \mathrm{~h}$ and f) $140 \mathrm{~h}$.

Figure 5. Mean surface roughness at various hydrothermal degradation times for all samples.

Figure 6. Nomarski microscopy images of exposed surface after $140 \mathrm{~h}$ of LTD for a) LAVAMW, b) LAVA-CS, c) LAB-MW and d) LAB-CS.

Figure 7. FE-SEM micrographs of the degraded zone profiles after $140 \mathrm{~h}$ of LTD for a) LAVAMW, b) LAVA-CS, c) LAB-MW and d) LAB-CS.

Figure 8. Young's modulus and hardness values as a function of LTD exposure time of all samples.

Figure 9. Young's modulus as a function of indenter penetration depth profile for a) LAB-MW and b) LAB-CS after different exposure times to LTD. 